\title{
Vegetable oils extraction of polycyclic aromatic hydrocarbons from an Algerian quagmire
}

\author{
Berrabia Nadjet ${ }^{1}$, Pemanos Yelda Bakoz ${ }^{2}$, Gherbi Abdellah ${ }^{1}$, Hamada Boudjema ${ }^{1}$ * \\ ${ }^{1}$ Department of chemistry, University M'hamed Bougara of Boumerdes, Boumerdes, Algeria \\ ${ }^{2}$ Depatment of Chemistry, College of Science, University of ZaKho, Kurdistan Rigion, Iraq
}

Email address:

bou.hamada@yahou.fr (H. Boudjema)

\section{To cite this article:}

Berrabia Nadjet, Pemanos Yelda Bakoz, Gherbi Abdellah, Hamada Boudjema. Vegetable Oils Extraction of Polycyclic Aromatic Hydrocarbons from an Algerian Quagmire. American Journal of Applied Chemistry. Vol. 2, No. 1, 2014, pp. 6-9.

doi: 10.11648/j.ajac.20140201.12

\begin{abstract}
Polycyclic aromatic hydrocarbons (PAHs) are carcinogenic substances which are resistant to environmental degradation due to their high hydrophobic nature. Soil contaminated with PAHs pose potential risks to human and ecological health, therefore concern over their adverse effects have resulted in extensive studies on their removal from contaminated soils. The main propose of this work was to extract this pollutants from two real quagmire samples from the region of Hassi Messaoud (Algeria), using three kinds of vegetable oils (Peanut oil, rapeseed oil, and coconut oil) which are naturals, cost effectives, and biodegradables extractors at two different temperatures $\left(30\right.$ and $\left.70{ }^{\circ} \mathrm{C}\right)$. Three PAHs that are representing of their present in contaminated quagmire were characterized using IR-FTIR and HPLC.
\end{abstract}

Keywords: PAHs, Extraction, Quagmire, Soil Rehabilitation

\section{Introduction}

Polycyclic aromatic hydrocarbons (PAHs) are ubiquitous environmental contaminants generated by the incomplete combustion of organic materials such as coal, wood, oil, gas and garbage. They belong to the group of per-sistent organic pollutants (POPs), which, according to the protocol on POPs established in 1998 in Denmark, is characterized with toxic, persistent, bio accumulative properties as well as long-range transboundary atmospheric transport and deposition with negative effects on environmental and human health [1]. known for its mutagenic and carcinogenic effect. They are generally more resistant to biodegradation than many saturated biomarkers and tend to persist in contaminated sediments, water, soil, atmospheric particulate matter and some organisms such as mussels [7].

(PAHs) constitute a group of priority pollutants, which are present in the soils of many industrially contaminated sites, particularly those associated with the petroleum industry. The majority of these substances are highly persistent, easily adsorb onto the organic matter of solid particles, forming persistent micropollutants in the environment, less degradable chemical forms because of their very low water solubility, their intrinsic chemical stability, and high recalcitrance to degradation [2].

Residual PAH containing material, either mixed in soils or sediments or occurring as a discrete phase, can also lead to the long-term desorption and dissolution of PAHs into groundwater. Therefore, the cleanup of these contaminated sites is an urgent task [3]. A variety of physical, chemical and biological remediation techniques have been proposed and have been demonstrated to have varying degrees of success [9] one commonly employed soil remediation technology is soil washing or solvent extraction. At bench scale solvent washing treatments of PAH-contaminated soil, various solvent mixtures including cyclohexane: ethanol mixture $(3: 1, \mathrm{v} / \mathrm{v})$, dichloromethane, ethanol and methanol have been tested significantly high volumes of solvents and surfactants reaching up to solvent: soil ratios of 100:1 (v/w) are necessary to remove highly contaminated soil effectively.[4]

The solvents used, for example, alkanes, alcohols, ketones and alkylamines are typically flammable, volatile and toxic and their use involves significant safety and environmental risks and application of stringent costly measures to minimize those risks. Efficiency of extraction is influenced by a variety of factors including temperature, $\mathrm{pH}$, and duration of extraction and solvent volume [5].

A search for less hazardous solvents for use in extraction 
of hydrophobic contaminants lead us to consider vegetable oil as a natural nontoxic, inexpensive, and effective extractant for this application. [5,3] It has also been demonstrated that vegetable oil amendment increased the biodegradation of various PAHs by $15-80 \%$ in PAHcontaminated weathered soil slurry systems. Vegetable oil in soil can be rapidly degraded if optimal microbial conditions are well maintained [3]. The use of vegetable oil in Fenton's chemistry for the rehabilitation of PAH-contaminated soils is also reported by Bogan et al. (2003) [6].

In this work three kinds of vegetable oils (rapeseed oil, peanut oil and coconut oil) were used as an alternative to solvent extraction of three PHAs (acenaphthene, fluoranthene and phenanthrene) from quagmire of Hassi Messaoud region (Algeria) at two different temperatures ( 30 and $70{ }^{\circ} \mathrm{C}$ ). Qualitative analysis (IR, UV-VIS and HPLC) were be realised on all samples to compare the effect of temperature and nature of oil on the qualitative extraction. A comparison between oil extraction and soxlet extraction using methanol was also realised.

\section{Experimental}

\subsection{Samples}

Two samples of contaminated quagumir A and B Fig.1 were collected at Hassi Messaoud field (southeast Algeria) a region seriously affected by flushing fluids. Appreciable levels of some of the analytes were therefore anticipated. Humidity of samples evaluated gravimetrically, after drying samples in an oven at $105{ }^{\circ} \mathrm{C}$ for $2 \mathrm{~h}$ it was $55,55 \%$ for sample A and 43,74\% for sample B. The humidity of samples $\mathrm{w}$ the total Carbone, nitrogen, and sulphur contents of the quagmires were $10,7 \%, 2,4 \%, 0,79 \%$ respectively, for sample A, and $6,88 \%, 1,8 \%, 0,57 \%$ for sample B.

\subsection{Chemicals and Reagents}

PAHs, acenaphthene (ACE) (purity: 97\%), fluoranthene (FLA) (purity: 98.5\%), phenanthrene (PHE) (puriy: 98\%) were acquired from Fluka. Methanol was purchased from Panreac (purity: residue analysis), anhydrous sodium sulphate (purity: pro analysis) and octadecyl-bonded silica (RP-C18) from Sigma-Aldrich. vegetable oils (rapeseed oil, peanut oil, coconut oil).

Stock standard solutions of each PAH at a concentration of $1 \mathrm{mg} / \mathrm{ml}$ were prepared in methanol and stored at $4{ }^{\circ} \mathrm{C}$ in the dark.

\subsection{Apparatus}

IR analyses were realized with IR-FTIR spectrophotometer Agilent.

HPLC analyses were realized with JASCO chromatographic system.

\subsection{Soxhlet Extraction}

Soxhlet extraction was performed using $5 \mathrm{~g}$ portion of soil to which $5 \mathrm{~g}$ of anhydrous sodium sulfate was added. The mixture was transferred into a clean filter paper and into a Soxhlet assembly fitted with a $100 \mathrm{~mL}$ flask [8]. A $100 \mathrm{~mL}$ of methanol was added and the whole assembly was heated for $15 \mathrm{~h}$. After extraction, the extracts were concentrated by means of a rotary evaporator to a volume of approximately $5 \mathrm{ml} \mathrm{[10].}$

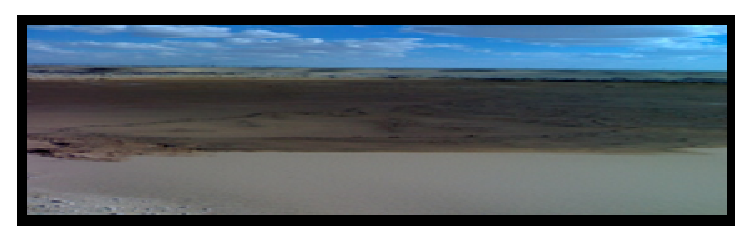

Quagmire A

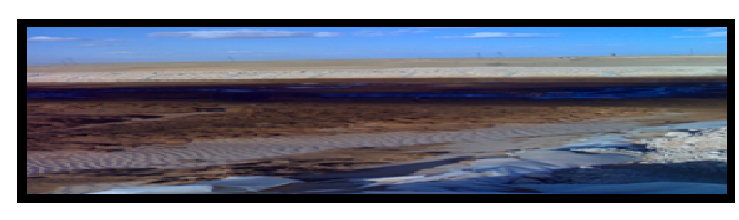

Quagmire B

Figure 1. Photography of the two quagmires.

\subsection{Extraction with Vegetable Oils}

Extractions were performed in $50 \mathrm{ml}$ glass vials with

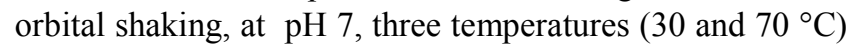
duration was $24 \mathrm{~h}$ and the ratio quagmire : oil was 10:10 (w:v). After extraction the oil was separated with simple filtration and here volume was measured to calculate oil recovery.

\subsection{Preparation of Oil Samples for Analysis}

Contaminated oil samples were extracted with methanol and analyzed by IR and HPLC-UV.

\subsection{HPLC Analysis}

PAH analysis was performed with an HPLC equipped with a gradient pump (Jasco PU 980), a manual sampler, and a reverse-phase C- 18 column an UV-vis detector (Jasco UV-2075). The elution system was acetonitril/eau 60:40 (v : v). Separation was done at room temperature using a constant flow-rate of $1,5 \mathrm{ml} / \mathrm{min}$ and the mobile phase was degassed during analysis by using an helium stream. $8 \mu \mathrm{l}$ of sample was injected into the HPLC by the manual sampler. An external standard (prepared by dilution of standard solutions) were used for qualification of the 3 PAHs.

\section{Results and Discussion}

\subsection{IR Results}

The results of the IR analysis represented in the Fig. 2, Fig. 3, Fig. 4 shows that the IR spectra of the three standards are similar in that the three standards have the 
same structure, which involved the same vibration. The standards have 1. An absorption band at $3000 \mathrm{~cm}-1$ corresponding to the $\mathrm{CH}$ stretching vibration according to Tipson and al (1971) and aromatic CH (3075 and

$3030 \mathrm{~cm}-1)$ according to Parker (1971). 2. Peaks located between 1450 and $1400 \mathrm{~cm}^{1}$ corresponding to the vibration of the backbone of the compounds with three condensate aromatic rings (anthracenes). 3. A band between 1100 and 1000 corresponds to the bending vibration in the plane of $\mathrm{CH}$ aromatics.

The absorption bands located between 900 and $700 \mathrm{~cm}^{-1}$ are characteristic of the bending vibrations of the condensate aromatic rings substituted. Values between 810 and $785 \mathrm{~cm}^{-1}$ are recorded in the literature for this kind of compounds [11].

By comparing the IR spectra of sloughs A and B respectively shown in fig2 and fig4, we note that the IR spectrum of quagmire A includes peaks observed in all the spectra of standards, which confirms the presence of these compounds in the extract.

In the spectrum of quagmire $B$, we note the absence of absorption peaks of most standards. This is probably due to the absence or low (PAHs) content in this quagmire B which is reinforced by the low organic matter contained in this quagmire $(0.8 \%)$ against $6 \%$ for $\mathrm{A}$.

The same absorption bands are observed in the IR spectra of extracts from the quagmire A at 30 and $70 \mathrm{C}^{\circ}$ (Fig. 2 and Fig. 3 respectively). So temperature have not influence on extraction of polyaromatic compounds contained in the quagmire.

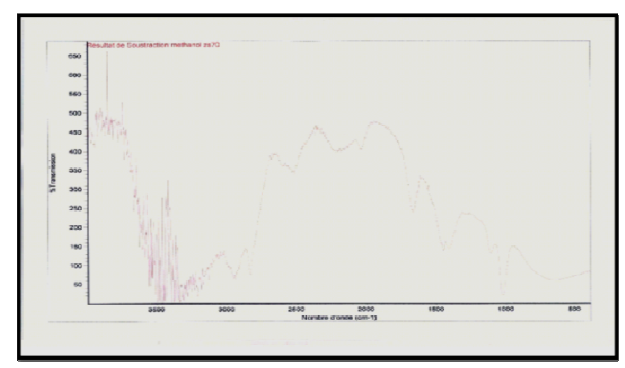

Figure 2. IR spectrum of sample extract at $30 C^{\circ}$ (quagmire A).

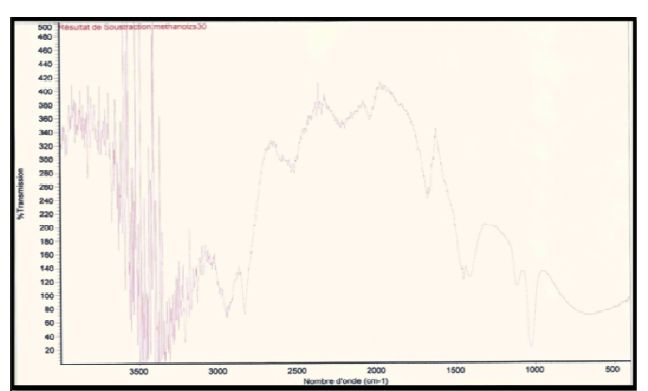

Figure 3. IR spectrum of sample extract at $70 C^{\circ}$ (quagmire A).

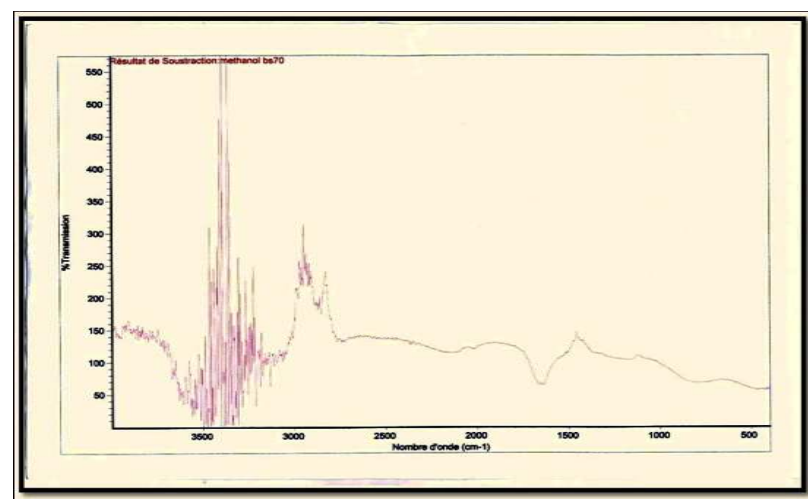

Figure 4. IR spectrum of sample extract at $70 \mathrm{C}^{\circ}$ (quagmire B).

\subsection{HPLC Results}

Results of HPLC-UV analysis represented in figures fig5, fig6, fig fig7 inform us that according to the chromatograms of standards, their corresponding peaks are well separated which facilitates their detection in the chromatogram of the mixture, ACE ( $\mathrm{tr}=7.5 \mathrm{~min}$.), FLA ( $\mathrm{tr}$ $=14 \mathrm{~min}$.), PHE (tr $=11 \mathrm{~min}$.). In the chromatograms (Fig. 6 and Fig. 5) corresponding to extracts obtained by peanut and rapeseed oils respectively, we note that the presence of characteristic peaks of the three studied (PAHs) which confirms their extraction by the two oils mentioned.

We observe on the chromatogram corresponding to the coconut oil Fig. 7 the absence of peaks corresponding to fluoranthene and phenanthrene so we can say that the qualitative extraction of (PAHs) is best with unsaturated vegetable oils.

We note that phenanthrene is represented by a lowintensity peak in the chromatograms on fig5 and fig6 whatsoever for samples drawn by vegetable oil or device soxlhet implying that it is presented in the quagmire sample A with a small amount.

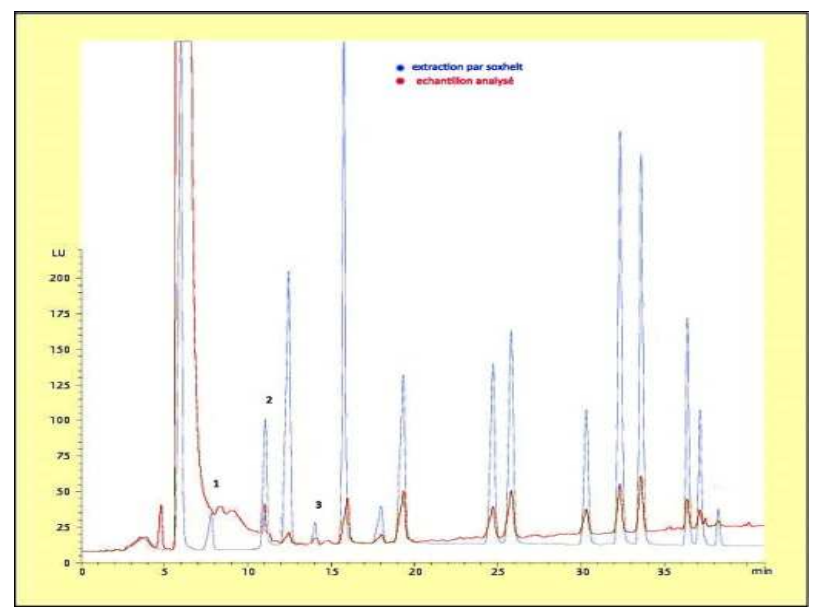

Figure 5. Chromatogram of the sample extracted with peanut oil. 


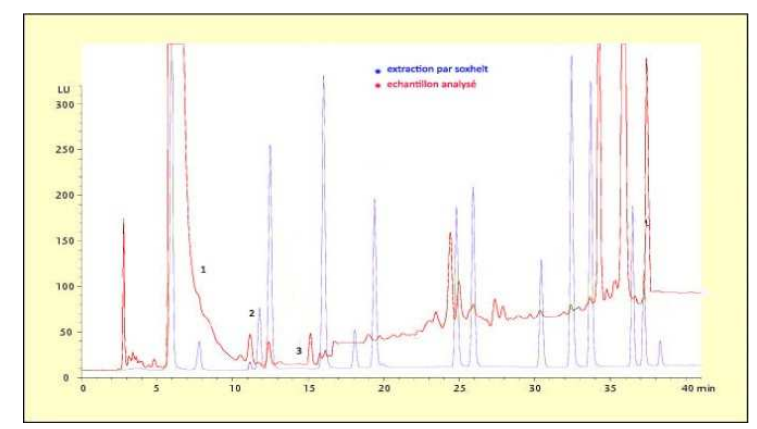

Figure 6. Chromatogram of the sample extracted with rapeseed oil.

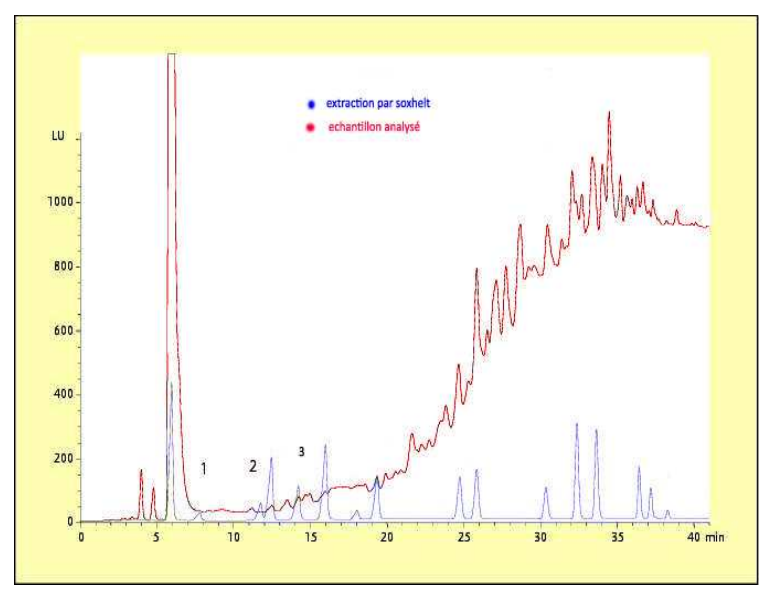

Figure 7. Chromatogram of the sample extracted with coconut oil.

\section{Conclusion}

The results showed that, the extraction of (PAHs) by vegetable oil from polluted quagmire is feasible at a temperature greater than or equal to $30 \mathrm{C}^{\circ}$, polyaromatic compounds can be extracted only with unsaturated vegetable oils and finally from a qualitative standpoint, the temperature does not effect on the results of extraction.

\section{References}

[1] Y. P. Guiavarc’h, A. Chahin, M. A. Dziurla, H. Toussaint, C. Feidt, and G. Rychen, "Evaluation of small dairy ruminant exposure to polycylic aromatic hydrocarbons: A biomarker approach"Smal. Rum. Res. Vol. 91, pp. 141 152, February 2010.
[2] M.A. Amezcua-Allieri, M.A. Ávila-Chávez , A. Trejo, and J. Meléndez-Estrada, "Removal of polycyclic aromatic hydrocarbons from soil: A comparison between bioremoval and supercritical fluids extraction", Chemosphere vol. 86, pp. 985-993, November 2012.

[3] Z. Gong, B. M. Wilke, K. Alef, P. Li, "Influence of soil moisture on sunflower oil extraction of polycyclic aromatic hydrocarbons from a manufactured gas plant soil" Scien. of the Tot. Envir. Vol. 343, pp. 51- 59, October 2005.

[4] E. V. Lau, S. Gan, H. K Ng, and P. E. Poh, "Extraction agents for the removal of polycyclic aromatic hydrocarbons (PAHs) from soil in soil washing technologies", Envir. Pol., vol. 184, pp. 640-649, September 2014.

[5] J. K. Pannu, A. Singh, and O. P. Ward, "Vegetable oil as a contaminated soil remediation amendment: application of peanut oil for extraction of polycyclicaromatic hydrocarbons from soil", Proc. Biochem., Vol. 39, pp. 1211-1216, Jun 2003.

[6] B. W. Bogan, V. Trbovic, and J. Robert Paterek, "Inclusion of vegetable oils in Fenton s chemistry for remediation of PAH-contaminated", Chemosphere, vol. 50, pp. 15-21, August 2002.

[7] O. L. G. Maioli, K. C. Rodrigues, B. A. Knoppers, and D. A. Azevedo, "Polycyclic aromatic and aliphatic hydrocarbons in Mytella charruana, a bivalve mollusk from Mundaú Lagoon, Brazil", Microchem. Jour., vol. 96, pp. 172-179, March 2010.

[8] W. Wang, B. Meng, X. Lu, Y. Liu, and S. Tao, "Extraction of polycyclic aromatic hydrocarbons and organochlorine pesticides from soils: A comparison between Soxhlet extraction, microwave-assisted extraction and accelerated solvent extraction techniques", analytica chimica acta, vol. 60 2, pp. 211-222, September 2007.

[9] L. Rehmann, G. P. Prpich, and A. J. Daugulis, "Remediation of PAH contaminated soils: Application of a solid-liquid two-phase partitioning bioreactor", Chemosphere, vol. 73, pp. 798-804, June 2008.

[10] M. Gfrerer, M. Serschen, and E. Lankmayr, "Optimized extraction of polycyclic aromatic hydrocarbons from contaminated soil samples", J. Biochem. Biophys. Methods, vol. 53, pp. 203-216, December 2002.

[11] B. Wojtkowiak, and M. Chabanel, "Spectrochimie Moleculaire", Editon Technique et documentation, 1957. 